\title{
Bathymetric curve (75 years old) validation, using the soil erosion transportation at Cuitzeo Lake Watershed
}

\author{
J. Lafragua ${ }^{1}$, A. Gutiérrez ${ }^{1}$, A. Bahena ${ }^{1}$, G. Moriel $^{2}$ \\ \& S. Férnández ${ }^{2}$ \\ ${ }^{1}$ Mexican Institute of Water Technology, Mexico \\ ${ }^{2}$ Secretary of Communications and Transports, Michoacán Center, \\ Mexico
}

\begin{abstract}
A new main road called Copandaro-La Cinta was built in the state of Michoacán, México; with a length of seventeen kilometers this road crosses Lake Cuitzeo (the section under study). In order to preserve the hydrological regime of this water area, the road structure was built with twenty sewers and four boat passages. With a $4000 \mathrm{~km}^{2}$ basin area, Lake Cuitzeo's watershed transports $1,296,461$ tons of sediment per year. Due to this great amount of material, a hydrological study was carried out. A hydraulic study was prepared including elevation-storage and elevation-area relationship curves since 1930. Using a longitudinal profile of the study section from April 2003, several bank levels are used to make an adjustment between the bathymetric curve and longitudinal profile, fixing some main structures points. In order to select the best alternative (to verify hydraulic works dimensions), soil erosion in the watershed is calculated using the Universal Soil Loss Equation (USLE); a sediment yield ratio is also obtainable. The results show that only 7 percent of the soil loss computed by the USLE appears as sediment yield in the watershed outlet. In the alternative selected, this quantity represents approximately $19 \mathrm{~cm}$ of sediment near the study section. With this alternative, the zero level in the longitudinal profile and in the bathymetric curve is at an elevation of 1823.34 and 1818.00 masl, respectively. Thus, all points of the bathymetric curve were adjusted at $5.34 \mathrm{~m}$. Flow routing through Lake Cuitzeo shows that the structures hydraulic capacity was enough to allow free water flow.
\end{abstract}

Keywords: soil erosion, hydrologic routing, inflow hydrographs, bathymetric curve, Lake Cuitzeo. 


\section{Introduction}

The new Copandaro-La Cinta main road on the Morelia-Salamanca freeway crosses the western area of Lake Cuitzeo. This structure has twenty sewers and four boat passages, and a bridge called Dren La Cinta. In previous years, this bridge allowed the passage of flows from Lake Cuitzeo to Lake Yuriria. To check the dimensions of the existent hydraulic works and verify their proper hydraulic operation it is necessary to consider the volumes of inflow for different return periods. Lake Cuitzeo's basin is divided into 25 sub-regions Lafragua et al., [6]. To carry out the hydraulic flow analysis, a bathymetric curve fundamental; however, the only one available was for the year 1930 (provided by the National Water Commission, Conagua). The objective of this study is to correct the bathymetric curve, taking into account the longitudinal profile of the road (2003), provided by the Secretariat of Communications and Transport (SCT). The amount of sediment deposited in the Lake is also required.

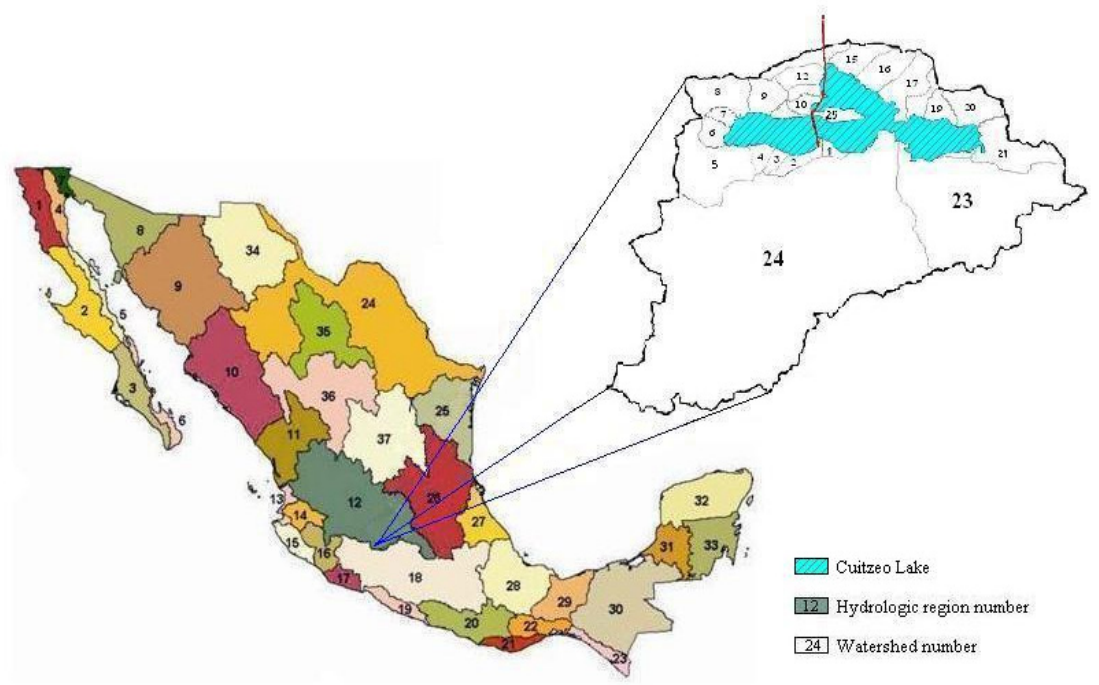

Figure 1: $\quad$ Location map of the study site.

\section{Study area}

Cuitzeo's watershed is located in the central part of the state of Michoacan, Mexico, between the coordinates $19^{\circ} 24^{\prime}$ to $20^{\circ} 05^{\prime}$ north latitude and $100^{\circ} 41^{\prime}$ to $101^{\circ} 33^{\prime}$ west longitude, and is part of hydrologic region number 12 called Lerma-Santiago, figure 1. Total watershed surface is $4,000 \mathrm{~km}^{2}$, of which 409.82 $\mathrm{km}^{2}$ of the Lake include hydrophyte vegetation and open water. The main river is Rio Grande of Morelia, with a drainage area of 2,043 square kilometers. The climate is temperate and subhumid with summer rainfall. The annual mean 
temperature ranges from $13^{\circ} \mathrm{C}$ to $20^{\circ} \mathrm{C}$, and mean annual precipitation is 804.0 millimeters. The Copandaro-La Cinta new main road crosses Lake Cuitzeo along seventeen kilometers (section in study) and has twenty sewers and four boat passages, in addition to La Cinta drain, figure 2.

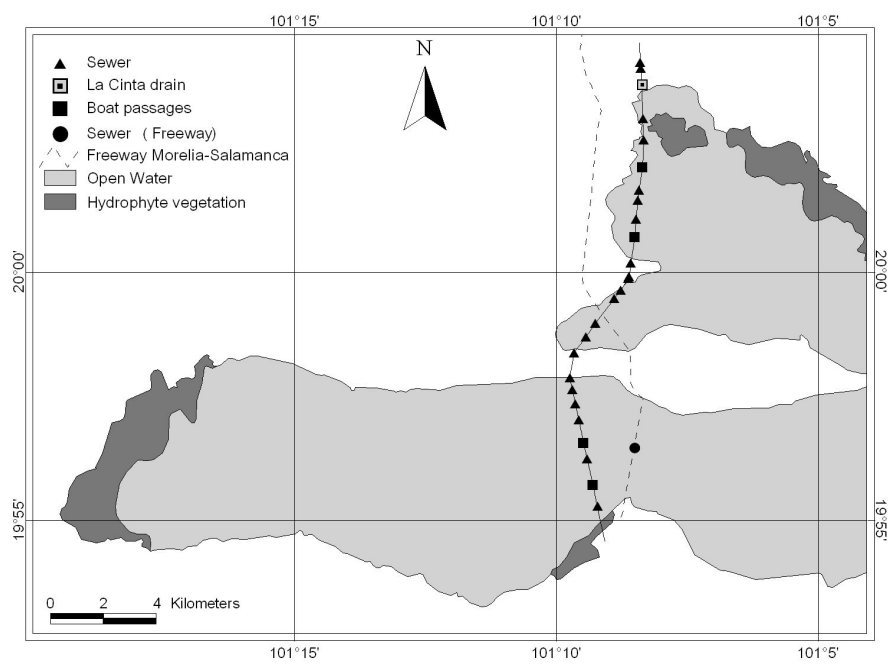

Figure 2: Hydraulic works location.

\section{Procedure description}

A bathymetric adjustment was conducted because there is no common level between the bathymetric curve (Conagua curve) and the longitudinal profile (SCT profile) of the study section. The procedure was as follows:

a) Drawing a longitudinal profile of the study section with the bathymetric curve information (Conagua Profile).

b) Analyzing possible alternatives of elevation similarities between the Conagua profile and the SCT profile.

c) Selecting the best alternative, by using the erosion value of the watershed outlet.

In order to calculate the annual erosion of Lake Cuitzeo's watershed, the Universal Soil Loss Erosion (USLE) equation was used, Ponce [7], given by

$$
A=R K L S C P
$$

where $A$ is the annual mean soil loss in tons $\mathrm{ha}^{-1}, R\left(\mathrm{MJ} \mathrm{mm} \mathrm{ha}^{-1} \mathrm{~h}^{-1}\right)$ is the rainfall erosivity index, calculated according to Figueroa et al. [4], $K$ (tons ha $\mathrm{h}$ $\mathrm{ha}^{-1} \mathrm{MJ}^{-1} \mathrm{~mm}^{-1}$ ) is the soil erodibility factor, calculated according to the FAO [3] methodology, $C$ is the cover and management factor, and $P$ is the support practice factor, assumed as 1.0, Izurieta et al. [5]. $L$ is the slope length factor 
and $S$ is the slope gradient, both are known as topographic factors and were calculated according to Izurieta et al. [5].

$$
L=\left(\frac{\lambda}{22.1}\right)^{m}
$$

where $\lambda(\mathrm{m})$ is the slope length. For this study area, according to Cortes [1], value $\lambda$ can be considered as $100 \mathrm{~m}$ and value $m$ is the slope-dependent dimension factor, considered as 0.4 .

$$
\begin{aligned}
& S=10.8 \operatorname{sen}(s g)+0.3 \text { with } s<9 \% \\
& S=16.8 \operatorname{sen}(s g)-0.5 \text { with } s>9 \%
\end{aligned}
$$

where $s g$ and $s$ are the slope of the land expressed in degrees and in percent, respectively.

With eqn (1), the amount of solid material eroded due to rainfall in one year is estimated; however, the total volume of material is not necessarily arriving at the watershed outlet. Escalante [2] declares that there are differences between soil loss in the watershed and the amount of sediment that the watershed outlet is receiving, and suggests using the following equation:

$$
A S=D R A
$$

where $A S$ (tons $\mathrm{ha}^{-1}$ ) is sediment in the watershed outlet, $A$ is the value calculated by eqn (1), and $D R$ is the sediment yield ratio, calculated by,

$$
D R=0.417662 A c^{-0.134958}-0.127097
$$

where $A c\left(\mathrm{mi}^{2}\right)$ is the watershed area.

Table 1: $\quad$ Fixed points.

\begin{tabular}{|c|c|l|c|}
\hline Alternative & $\begin{array}{l}\text { Point } \\
\text { number }\end{array}$ & \multicolumn{1}{|c|}{ Characteristic } & $\begin{array}{c}\text { Elevation difference (m) } \\
\text { (SCT minus Conagua) }\end{array}$ \\
\hline A-1 & 5 & $\begin{array}{l}\text { High point and located } \\
\text { outside the Lake. }\end{array}$ & 5.83 \\
\hline A-2 & 41 & High point. & 4.43 \\
\hline A-3 & 47 & Without major inflow. & 5.70 \\
\hline A-4 & 27 & $\begin{array}{l}\text { High point and without } \\
\text { major inflow. }\end{array}$ & 5.34 \\
\hline A-5 & 49 & $\begin{array}{l}\text { High point and without } \\
\text { major inflow. }\end{array}$ \\
\hline
\end{tabular}

\section{Results and discussion}

Using the Conagua elevation curve, 55 points were selected, including the 25 hydraulic works, to draw the longitudinal profile of the new main road. Many alternatives were analyzed by fixing points where erosion was not observed through time. Table 1 shows five alternatives indicating the fixed point 
considered, and table 2 shows the differences between the Conagua profile and the SCT profile, the rest of the alternatives showed a similar behavior.

Table 2: $\quad$ Selected points and elevation differences.

\begin{tabular}{|c|c|c|c|c|c|c|c|c|}
\hline No. & $\begin{array}{c}\text { Hydraulic } \\
\text { work }\end{array}$ & $\begin{array}{c}\text { Elevation } \\
\text { (SCT) }\end{array}$ & $\begin{array}{c}\text { Elevation } \\
\text { (Conagua) }\end{array}$ & A-1 & A-2 & A-3 & A-4 & A-5 \\
\hline 1 & & 1827.18 & 1820.50 & -0.85 & -2.25 & -0.98 & -1.34 & -1.41 \\
\hline 2 & & 1826.01 & 1820.00 & -0.18 & -1.58 & -0.31 & -0.67 & -0.74 \\
\hline 3 & & 1825.63 & 1819.50 & -0.30 & -1.70 & -0.43 & -0.79 & -0.86 \\
\hline 4 & & 1824.80 & 1819.00 & 0.03 & -1.37 & -0.10 & -0.46 & -0.53 \\
\hline 5 & & 1824.33 & 1818.50 & 0.00 & -1.40 & -0.13 & -0.49 & -0.56 \\
\hline 6 & & 1823.87 & 1818.00 & -0.04 & -1.44 & -0.17 & -0.53 & -0.60 \\
\hline 7 & & 1823.86 & 1818.00 & -0.03 & -1.43 & -0.16 & -0.52 & -0.59 \\
\hline 8 & & 1823.81 & 1818.00 & 0.02 & -1.38 & -0.11 & -0.47 & -0.54 \\
\hline 9 & & 1823.83 & 1818.00 & 0.00 & -1.40 & -0.13 & -0.49 & -0.56 \\
\hline 10 & & 1823.88 & 1818.50 & 0.45 & -0.95 & 0.32 & -0.04 & -0.11 \\
\hline 20 & & 1824.38 & 1820.50 & 1.95 & 0.55 & 1.82 & 1.46 & 1.39 \\
\hline 21 & & 1824.48 & 1820.50 & 1.85 & 0.45 & 1.72 & 1.36 & 1.29 \\
\hline 22 & & 1824.76 & 1820.00 & 1.07 & -0.33 & 0.94 & 0.58 & 0.51 \\
\hline 23 & & 1824.93 & 1819.50 & 0.40 & -1.00 & 0.27 & -0.09 & -0.16 \\
\hline 24 & & 1824.56 & 1819.00 & 0.27 & -1.13 & 0.14 & -0.22 & -0.29 \\
\hline 25 & & 1824.59 & 1819.00 & 0.24 & -1.16 & 0.11 & -0.25 & -0.32 \\
\hline 26 & & 1824.66 & 1819.00 & 0.17 & -1.23 & 0.04 & -0.32 & -0.39 \\
\hline 27 & & 1824.85 & 1819.51 & 0.49 & -0.91 & 0.36 & 0.00 & -0.07 \\
\hline 28 & & 1825.15 & 1819.86 & 0.54 & -0.86 & 0.41 & 0.05 & -0.02 \\
\hline 29 & & 1825.85 & 1820.00 & -0.02 & -1.42 & -0.15 & -0.51 & -0.58 \\
\hline 30 & & 1825.59 & 1820.50 & 0.74 & -0.66 & 0.61 & 0.25 & 0.18 \\
\hline 40 & $\mathrm{VC} 10$ & 1824.40 & 1819.62 & 1.05 & -0.35 & 0.92 & 0.56 & 0.49 \\
\hline 41 & $\mathrm{VC} 11$ & 1824.55 & 1820.12 & 1.40 & 0.00 & 1.27 & 0.91 & 0.84 \\
\hline 42 & $\mathrm{VC} 13$ & 1824.85 & 1819.31 & 0.29 & -1.11 & 0.16 & -0.20 & -0.27 \\
\hline 43 & VC14 & 1825.05 & 1819.17 & -0.05 & -1.45 & -0.18 & -0.54 & -0.61 \\
\hline 44 & VC15 & 1824.45 & 1818.99 & 0.37 & -1.03 & 0.24 & -0.12 & -0.19 \\
\hline 45 & $\mathrm{VC} 16$ & 1824.35 & 1819.00 & 0.48 & -0.92 & 0.35 & -0.01 & -0.08 \\
\hline 46 & $\mathrm{VC} 17$ & 1824.60 & 1819.03 & 0.26 & -1.14 & 0.13 & -0.23 & -0.30 \\
\hline 47 & $\mathrm{VC} 18$ & 1825.30 & 1819.60 & 0.13 & -1.27 & 0.00 & -0.36 & -0.43 \\
\hline 48 & VC19 & 1824.80 & 1819.70 & 0.73 & -0.67 & 0.60 & 0.24 & 0.17 \\
\hline 49 & VC21 & 1825.24 & 1819.97 & 0.56 & -0.84 & 0.43 & 0.07 & 0.00 \\
\hline 50 & $\mathrm{VC} 22$ & 1825.24 & 1819.98 & 0.57 & -0.83 & 0.44 & 0.08 & 0.01 \\
\hline 51 & PL1 & 1823.85 & 1818.02 & 0.00 & -1.40 & -0.13 & -0.49 & -0.56 \\
\hline 52 & PL2 & 1823.85 & 1818.02 & 0.00 & -1.40 & -0.13 & -0.49 & -0.56 \\
\hline 53 & PL3 & 1824.60 & 1819.00 & 0.23 & -1.17 & 0.10 & -0.26 & -0.33 \\
\hline 54 & PL4 & 1824.85 & 1819.38 & 0.36 & -1.04 & 0.23 & -0.13 & -0.20 \\
\hline 55 & PEMEX & 1824.45 & 1819.10 & 0.48 & -0.92 & 0.35 & -0.01 & -0.08 \\
\hline
\end{tabular}

For each alternative, total sediment area was obtained by considering the differences shown in table 2. Figure 3 shows the sediment areas of alternative 4, of 34,800 to $45,504 \mathrm{~km}$. Assuming a uniform distribution along the longitudinal profile, an average sediment height is estimated, table 3 .

In table 3 , the negative values indicate that the fixed point was eroded; therefore, these alternatives were eliminated. The rest of the alternatives have an average (elevation) height of between 0.18 and $1.5 \mathrm{~m}$. Consequently, we compared water levels measured by Conagua and SCT during the construction of the road, obtaining an average difference of $5.18 \mathrm{~m}$. Otherwise, we considered the incrustation value designated by the $\operatorname{SCT}(0.60 \mathrm{~m})$, then the points with 
differences between 0 and $-0.60 \mathrm{~m}$ were counted. In alternatives 4 and 5 , more points, 30 and 32 points, respectively, were found. Therefore, alternatives 4 and 5 are good options. In order to select only one alternative, watershed outlet soil erosion was considered. The variable values for each watershed from eqn (1) are shown in table 4.

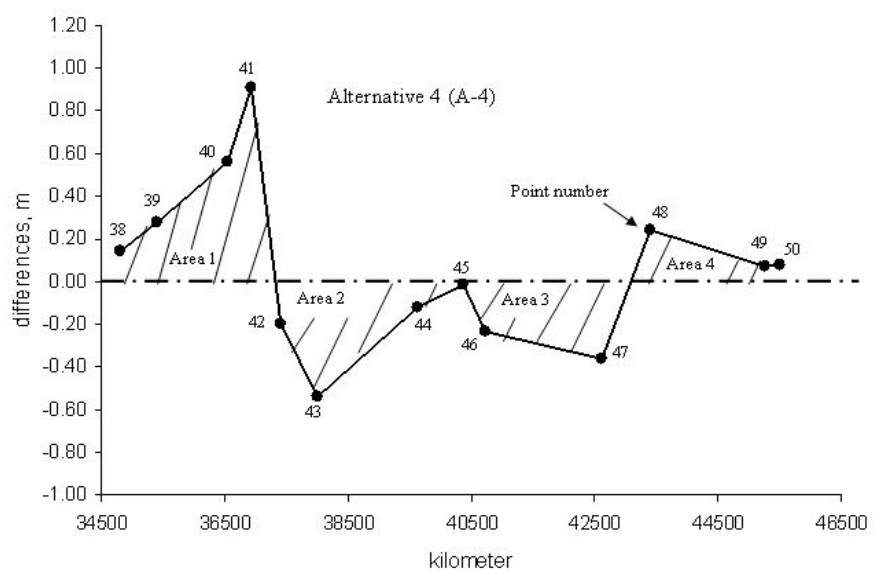

Figure 3: Sediment area along the new main road.

Table 3: $\quad$ Area and average sediment height.

\begin{tabular}{|c|c|c|}
\hline Alternative & $\begin{array}{c}\text { Total sediment } \\
\text { area, }\left(\mathrm{m}^{2}\right)\end{array}$ & $\begin{array}{c}\text { Average sediment } \\
\text { height, }(\mathrm{m})\end{array}$ \\
\hline A-1 & -5442.41 & -0.31 \\
\hline A-2 & 18784.97 & 1.08 \\
\hline A-3 & -3192.73 & -0.18 \\
\hline A-4 & 3037.17 & 0.18 \\
\hline A-5 & 4248.54 & 0.24 \\
\hline
\end{tabular}

The results of applying eqn (1) and eqn (2) are shown in the table 5. Soil loss in all watersheds in Lake Cuitzeo is $17,672,146$ tons year ${ }^{-1}$, but only 7 percent appears as sediment yield at the watershed outlet. Assuming that 1,296,461 tons of sediment arrived at the Lake per year in average, then in 75 years, the Lake has received $97 \mathrm{hm}^{3}$ of sediment. Due to unavailable information, we assume that the sediment is first deposited into the deeper areas, resulting in $70 \mathrm{~cm}$ of sediment in these areas and $19 \mathrm{~cm}$ approximately in the study area, figure 4. Finally, alternative 4 was selected because it exhibits the value closest to $19 \mathrm{~cm}$, obtained by calculating the erosion.

In the selected alternative, the zero level in the SCT and Conagua profiles is at an elevation of 1823.34 and 1818.00 masl, respectively, therefore, every point of the bathymetric curve was adjusted at $5.34 \mathrm{~m}$. Figure 5 shows the adjusted bathymetric curve. 
Table 4: $\quad$ USLE variables for each watershed.

\begin{tabular}{|c|r|r|r|r|r|r|r|r|}
\hline $\begin{array}{c}\text { Watershed } \\
\text { number }\end{array}$ & Area (ha) & $\begin{array}{c}\text { Rainfall } \\
(\mathrm{mm})\end{array}$ & $S(\%)$ & $R$ & $K$ & $L S$ & $C$ & $P$ \\
\hline 1 & 1194.11 & 705.79 & 17.0 & 2445.68 & 0.02 & 3.91 & 0.04 & 1.00 \\
\hline 2 & 2549.69 & 833.20 & 16.9 & 2883.03 & 0.02 & 4.09 & 0.13 & 1.00 \\
\hline 3 & 1293.56 & 845.52 & 16.6 & 2925.26 & 0.02 & 4.22 & 0.16 & 1.00 \\
\hline 4 & 2081.71 & 843.01 & 15.7 & 2916.70 & 0.02 & 3.81 & 0.20 & 1.00 \\
\hline 5 & 17517.45 & 802.72 & 13.0 & 2778.56 & 0.02 & 3.03 & 0.17 & 1.00 \\
\hline & & & & & & & & \\
\hline 14 & 4052.99 & 670.76 & 7.2 & 2325.29 & 0.02 & 1.41 & 0.34 & 1.00 \\
\hline 15 & 4862.76 & 625.53 & 6.1 & 2168.99 & 0.03 & 1.11 & 0.29 & 1.00 \\
\hline 16 & 5777.69 & 640.41 & 4.9 & 2220.35 & 0.03 & 0.83 & 0.23 & 1.00 \\
\hline 17 & 7207.63 & 715.52 & 10.2 & 2478.98 & 0.02 & 2.01 & 0.15 & 1.00 \\
\hline 18 & 2578.24 & 720.54 & 18.2 & 2496.25 & 0.02 & 5.09 & 0.24 & 1.00 \\
\hline 19 & 2898.54 & 720.54 & 17.6 & 2496.25 & 0.02 & 5.02 & 0.28 & 1.00 \\
\hline 20 & 6807.55 & 734.05 & 12.9 & 2542.42 & 0.03 & 3.16 & 0.29 & 1.00 \\
\hline 21 & 10859.16 & 784.39 & 13.7 & 2714.75 & 0.03 & 3.25 & 0.16 & 1.00 \\
\hline 22 & 707.42 & 742.85 & 16.4 & 2572.35 & 0.02 & 3.95 & 0.10 & 1.00 \\
\hline 23 & 55985.36 & 767.67 & 16.9 & 2657.86 & 0.03 & 4.47 & 0.21 & 1.00 \\
\hline 24 & 204294.24 & 860.61 & 12.2 & 2977.92 & 0.02 & 2.93 & 0.23 & 1.00 \\
\hline 25 & 2513.50 & 714.65 & 7.3 & 2476.29 & 0.02 & 1.68 & 0.42 & 1.00 \\
\hline & & & & & & & & \\
\hline
\end{tabular}

Table 5: $\quad$ Erosion results for each watershed.

\begin{tabular}{|r|r|r|r|r|r|r|r|}
\hline $\begin{array}{c}\text { Watershed } \\
\text { number }\end{array}$ & $\begin{array}{c}\text { Eqn. (1) } \\
\left(\text { tons ha }^{-1} \mathrm{yr}^{-1}\right)\end{array}$ & DR & $\begin{array}{c}\text { Eqn. (4) } \\
\left(\text { tons }^{-1}\right)\end{array}$ & $\begin{array}{c}\text { Watershed } \\
\text { number }\end{array}$ & $\begin{array}{c}\text { Eqn. (1) } \\
\left(\text { tons ha }^{-1} \mathrm{yr}^{-1}\right)\end{array}$ & DR & $\begin{array}{c}\text { Eqn. (4) } \\
\left(\text { tons }^{-1}\right)\end{array}$ \\
\hline 1 & 7.39 & 0.21 & 1877.97 & 14 & 27.47 & 0.16 & 17932.76 \\
\hline 2 & 28.19 & 0.18 & 12912.61 & 15 & 17.77 & 0.15 & 13312.72 \\
\hline 3 & 36.75 & 0.21 & 9940.18 & 16 & 10.67 & 0.15 & 9100.33 \\
\hline 4 & 39.67 & 0.19 & 15538.25 & 17 & 17.36 & 0.14 & 17454.16 \\
\hline 5 & 26.89 & 0.11 & 51531.12 & 18 & 67.23 & 0.18 & 31059.16 \\
\hline 6 & 18.06 & 0.17 & 9160.92 & 19 & 84.92 & 0.17 & 42924.66 \\
\hline 7 & 24.06 & 0.20 & 7189.23 & 20 & 67.41 & 0.14 & 64968.17 \\
\hline 8 & 33.96 & 0.13 & 39552.37 & 21 & 42.58 & 0.13 & 57874.83 \\
\hline 9 & 22.46 & 0.16 & 16011.22 & 22 & 25.87 & 0.24 & 4347.56 \\
\hline 10 & 128.75 & 0.23 & 25097.48 & 23 & 80.84 & 0.08 & 339784.28 \\
\hline 11 & 51.41 & 0.19 & 17831.81 & 24 & 46.85 & 0.04 & 408418.37 \\
\hline 12 & 101.81 & 0.20 & 33020.48 & 25 & 37.71 & 0.18 & 17084.97 \\
\hline 13 & 52.54 & 0.16 & 32535.29 & Total & 1098.61 & 0.16 & 1296460.88 \\
\hline
\end{tabular}

The adjusted bathymetric curve was used by routing floods with the storm design of 50 years. We consider tree scenarios: a) inflow from the east and west during the same time, figure $6, \mathrm{~b}$ ) only inflow from the east, and c) only inflow from west. The hydraulic results including the 25 hydraulic works located in the study site and the sewer located in the freeway are shown in table 6. Maximum elevation at the end inflow was 1824.53 masl from hydraulic work VC1 to VC5 and 1824.67 masl from VC10 to La Cinta drain. The maximum discharge obtained in the hydraulic works was $4.94 \mathrm{~m}^{3} \mathrm{~s}^{-1}$ and maximum velocity was 1.33 $\mathrm{m} \mathrm{s}^{-1}$. In the freeway sewer, the maximum discharge was $11.89 \mathrm{~m}^{3} \mathrm{~s}^{-1}$ and maximum velocity was $2.8 \mathrm{~m} \mathrm{~s}^{-1}$ Lafragua et al. [6]. 

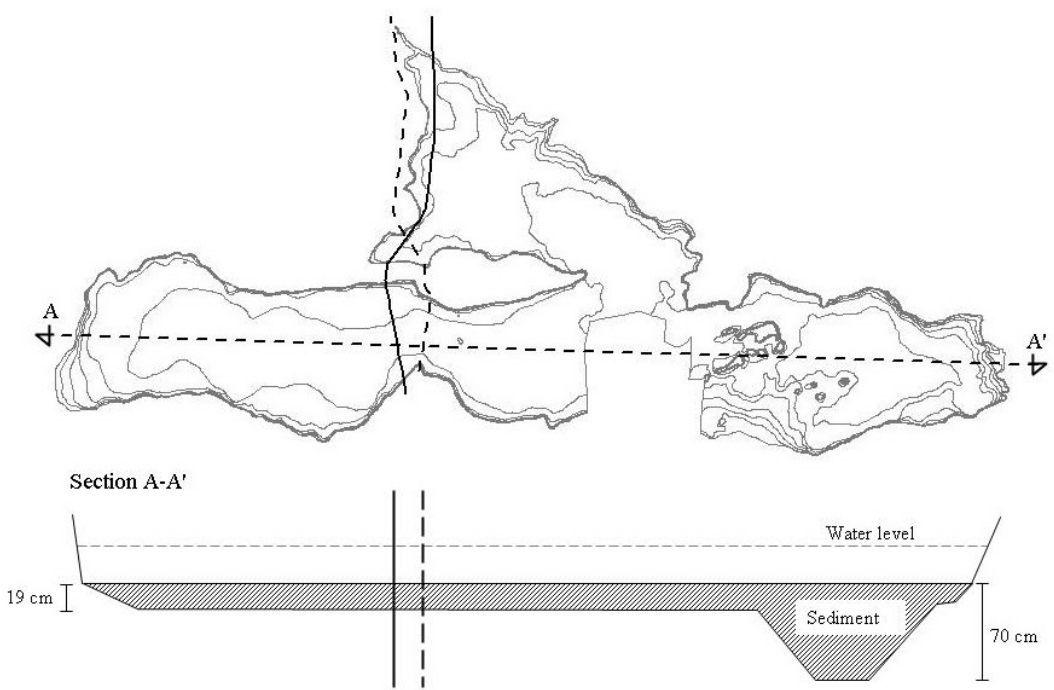

Figure 4: $\quad$ Scheme showing sediment deposit into Lake Cuitzeo.

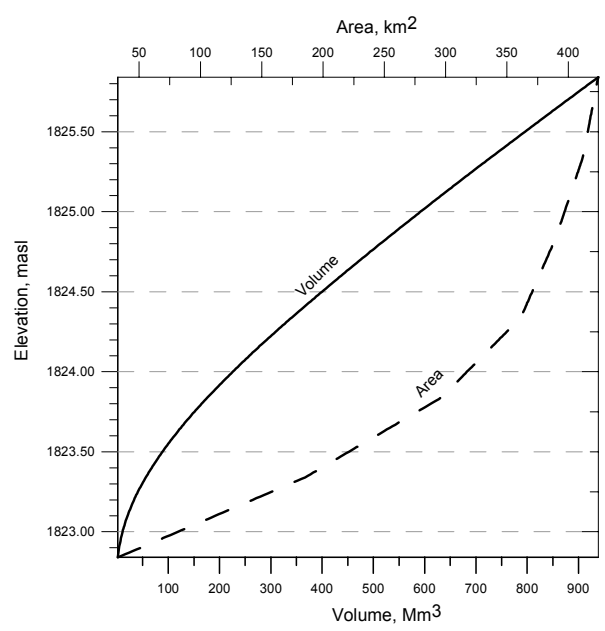

Figure 5: Adjusted bathymetric curve.

\section{Conclusions}

A bathymetric curve from 1930 was adjusted by using a longitudinal profile of the study section from 2003, and one sediment yield ratio was used in order to select the best alternative. All points of the bathymetric curve were adjusted at $5.34 \mathrm{~m}$. Also considered were water levels measured by SCT and Conagua during the building of the new main road, furthermore, $60 \mathrm{~cm}$ from incrustation designed by SCT was considered. 


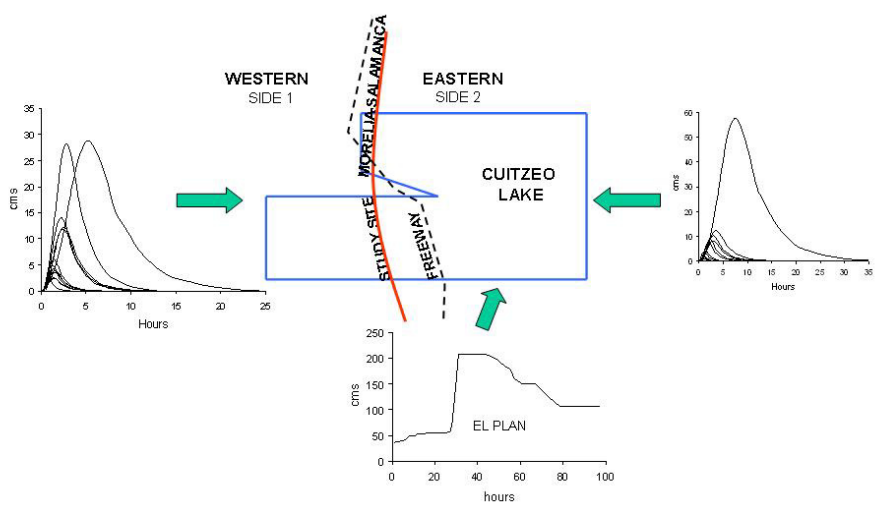

Figure 6: Input floods for rainfall (50-years).

Table 6: Hydraulic results.

\begin{tabular}{|c|c|c|c|c|c|c|c|c|c|}
\hline $\begin{array}{l}\text { Hydraulic } \\
\text { Work }\end{array}$ & $\begin{array}{c}\text { Footing } \\
\text { Elevation } \\
\text { (masl) }\end{array}$ & $\begin{array}{c}\text { Grade } \\
\text { Elevation } \\
\text { (masl) }\end{array}$ & $\begin{array}{c}\text { Initial } \\
\text { Elevation } \\
(\mathrm{m})\end{array}$ & $\begin{array}{c}\text { End } \\
\text { Elevation } \\
(\mathrm{m})\end{array}$ & $\begin{array}{c}\text { Initial } \\
\text { Depth } \\
(\mathrm{m})\end{array}$ & $\begin{array}{l}\text { End } \\
\text { Depth } \\
(\mathrm{m})\end{array}$ & $\begin{array}{l}\text { Area } \\
\left(\mathrm{m}^{2}\right)\end{array}$ & $\begin{array}{c}\text { Discharge } \\
\left(\mathrm{m}^{3} \mathrm{~s}^{-1}\right) \\
\end{array}$ & $\begin{array}{c}\text { Velocity } \\
\left(\mathrm{m} \mathrm{s}^{-1}\right)\end{array}$ \\
\hline $\mathrm{VC} 1$ & 1823.95 & 1826.60 & 1824.50 & 1824.53 & 0.55 & 0.58 & 1.10 & 0.42 & 0.38 \\
\hline PL1 & 1823.85 & 1829.75 & 1824.50 & 1824.53 & 0.65 & 0.68 & 20.38 & 4.71 & 0.23 \\
\hline $\mathrm{VC} 2$ & 1823.80 & 1826.60 & 1824.50 & 1824.53 & 0.70 & 0.73 & 1.40 & 0.58 & 0.42 \\
\hline PL2 & 1823.85 & 1829.65 & 1824.50 & 1824.53 & 0.65 & 0.68 & 20.38 & 4.94 & 0.24 \\
\hline $\mathrm{VC} 3$ & 1823.85 & 1826.60 & 1824.50 & 1824.53 & 0.65 & 0.68 & 1.31 & 0.41 & 0.32 \\
\hline $\mathrm{VC} 4$ & 1823.95 & 1826.60 & 1824.50 & 1824.53 & 0.55 & 0.58 & 1.11 & 0.44 & 0.40 \\
\hline VC9 & 1824.55 & 1826.30 & 1824.50 & 1824.71 & -0.05 & 0.16 & 0.30 & 0.30 & 1.03 \\
\hline $\mathrm{VC} 10$ & 1824.40 & 1826.30 & 1824.50 & 1824.67 & 0.10 & 0.27 & 0.35 & 0.41 & 1.15 \\
\hline PEMEX & 1824.45 & 1826.30 & 1824.50 & 1824.67 & 0.05 & 0.22 & 3.43 & 1.21 & 0.35 \\
\hline VC11 & 1824.55 & 1826.30 & 1824.50 & 1824.67 & -0.05 & 0.12 & 0.07 & 0.03 & 0.41 \\
\hline VC13 & 1824.85 & 1826.30 & - & - & - & - & - & - & - \\
\hline VC14 & 1825.05 & 1826.30 & - & - & - & - & - & - & - \\
\hline PL3 & 1824.60 & 1830.90 & 1824.50 & 1824.67 & -0.10 & 0.07 & 1.90 & 0.07 & 0.04 \\
\hline $\mathrm{VC} 15$ & 1824.45 & 1826.30 & 1824.50 & 1824.67 & 0.05 & 0.22 & 0.26 & 0.24 & 0.95 \\
\hline $\mathrm{VC} 16$ & 1824.35 & 1826.30 & 1824.50 & 1824.67 & 0.15 & 0.32 & 0.45 & 0.60 & 1.33 \\
\hline $\mathrm{VC} 17$ & 1824.60 & 1826.30 & 1824.50 & 1824.67 & -0.10 & 0.07 & 0.13 & 0.01 & 0.06 \\
\hline PL4 & 1824.85 & 1830.90 & - & - & - & - & - & - & - \\
\hline $\begin{array}{c}\text { LA } \\
\text { CINTA }\end{array}$ & 1823.70 & 1829.40 & 1824.50 & 1824.67 & 0.80 & 0.987 & 24.35 & 0.28 & 0.01 \\
\hline $\begin{array}{c}\text { Sewer } \\
\text { freeway } \\
(\mathrm{D}=5 \mathrm{~m})\end{array}$ & 1823.34 & & 1824.50 & 1824.64 & 1.16 & 1.30 & 4.13 & 11.89 & 2.88 \\
\hline
\end{tabular}


The adjusted bathymetric curve was used by routing floods with the storm design of 50 years. Flow routing through Lake Cuitzeo shows that hydraulic structures were enough to permit a free flow through them.

\section{References}

[1] Cortés T.H., (2005). Personal communication, 10 October 2005. Resarcher. Mexican Institute of Water Technology (IMTA).

[2] Escalante S.C., (2005). Capítulo 8. Efecto en la estimación del factor erosivo de la lluvia en el aporte de sedimentos. En: Rivera-Trejo F., Gutiérrez-López A., Val-Segura R., Mejía-Zermeño R., Sánchez-Ruiz P., Aparicio-Mijares J, Díaz-Flores L., (Editores). "LA MEDICIÓN DE SEDIMENTOS EN MÉXICO”. Ediciones IMTA-UJAT, México. 325 p. ISBN-968-5536-53-8.

[3] FAO, (1980). Metodología provisional para la evaluación de la degradación de los suelos. Roma. 86 p.

[4] Figueroa S.B, Amante O.A, Cortés T.H, Pimentel L.J, Osuna Ceja E.S, Rodríguez O.J.M and Morales F.F.J, (1991). Manual de predicción de pérdidas de suelo por erosión. Subdirección de Conservación del Suelo y Agua, SARH.

[5] Izurieta J., Huerto D.R., Medina M.R., Cortés T.H., Spillecke W.K.W., Brena Z.J. y Castillo R.C., (2002). Estimación del impacto de las cargas de contaminantes del Dren Zurumútaro en el lago de Pátzcuaro y propuestas de tratamiento. SGC-UAPS-MICH-02-006-RF-CC, CNAIMTA.

[6] Lafragua C.J., Gutiérrez L.A., Báhena H.A., Leal B.G., and Peña P.T., (2005). Dimensionamiento de alcantarillas y pasos de lancha, en el tramo carretero Copándaro-La Cinta, Morelia, Michoacán. IMTA-SCT. Proyecto TH-0550.

[7] Ponce V.M., (1989). Engineering Hydrology: Principles and Practices. Prentice Hall. 